\title{
The Bezier Curve Representation of Binary Phase Diagram
}

\author{
Hong Bao \\ School of Computer \& Communication Engineering \\ University of Science and Technology Beijing \\ BeiJing, China \\ e-mail: baohong@ies.ustb.edu.cn
}

\author{
Tian Hang \\ School of Computer \& Communication Engineering \\ University of Science and Technology Beijing \\ BeiJing, China \\ e-mail: 632224475@qq.com
}

\begin{abstract}
The phase diagram is a integrated graphic which represents the relationship among the state of material phase, temperature and composition. It can help the researchers to get the information about the change of material composition phase species at different temperature, the relative amount of each phase may occur and the changes when the temperature changes. So the phase diagram is known as the material design guide book, map of the metallurgical workers, and the source of thermodynamics data. In this paper, the main work is that the analysis of the characteristic of the graphic and the internal correlation of the data through the investigation and analysis of the existing methods of drawing phase diagram, combined with the phase diagram image and data, and then propose a method to represent the binary phase diagram based on Bezier curve which achieved a simple, efficient and accurate way to represent and store phase diagram.
\end{abstract}

Keywords-phase diagram; Bezier curve; control point; De Casteljau arithmetict

\section{INTRODUCTION}

The phase diagram is known as the material design guide book, map of the metallurgical workers, and the source of thermodynamics data, which provides the necessary physical and chemical data for selecting the composition of material, formulating the heat treatment process and processing technology. With the development of modern materials science and the application of Thermal analysis X-ray diffraction, Metallographic analysis and other testing methods, the field of studying the experimental phase diagram is expanding and it also greatly promote the development of the experimental phase diagram [1-6]. However, due to the workload, the limit of the experimental conditions and some material samples are hard to pretreatment, the precise mapping the phase diagram is still difficulty. Because of this, only a few countries have the condition to have their own thermodynamic database, the other countries that lack of thermodynamic database will be controlled by others when they are doing research on phase diagram. In summary, finding a simple, efficient, and accurate way to represent and store phase diagram is very important. The Bezier curve in computer graphic makes it possible.

Bezier curve is a curve that determined by its feature polygon (a polygon generated by the connection between control points), the properties of the Bezier curve is also described through the control points [7-8]. So the current Bezier curve algorithms all make the control points as premise. However, in this paper, we do it on the other way, we can use the points on the binary phase diagram as the online points on the Bezier curve, and the can use these points to get the control points belonged to the Bezier curve reversely, and finally, we can record the control points to describe a binary phases diagram.

\section{REPRESENTATION OF PHASE DiAgRAM}

The simplest phase diagrams are pressure-temperature diagrams of a single simple substance, such as water (Fig.1 shows the solid green line applies to most substances; the dotted green line gives the anomalous behavior of water. The green lines mark the freezing point and the blue line the boiling point, showing how they vary with pressure).The axescorrespond to the pressure and temperature. The phase diagram shows, in pressure-temperature space, the lines of equilibrium or phase boundaries between the three phases of solid, liquid, and gas.

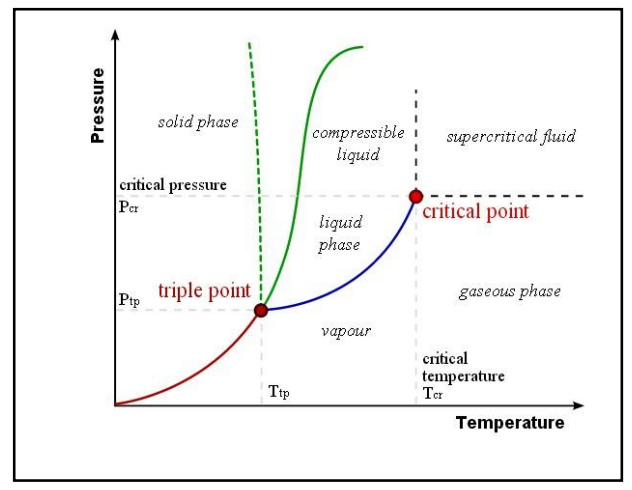

Figure 1. A typical phase diagram

Having introduced the phase diagram in general, let's talk about the representation of phase diagram, traditional methods, CALPHAD methods and curve fitting methods, the first two methods are based on the experimental data, and the last one is a supplement to the former.

\section{A. Traditional Method}

The phase diagrams generated by the tradition method (Dynamic method and Static method) are totally based the data obtained by the experimental method and painted by hand, and this kind of phase diagrams are almost stored in the handbooks about thermodynamics. When we want to use some phase diagrams we must look up the handbooks. But using the traditional method to make the phase diagram is a very delicate and difficult task, and the accuracy is also a big problem. What's more the hand-drawing diagrams also have the error 
made by man. In addition, the component of phase diagram is increasing as with the development of science and technology, the continuous development of new compounds and new system, the experimental determination of the phase diagram has lagged far behind the needs of the actual requirements.

\section{B. CALPHAD Method}

CALPHAD stands for Calculation of Phase Diagrams[1011]. An equilibrium phase diagram is usually a diagram with axes for temperature and composition of a chemical system. It shows the regions where substances or solutions (i.e. phases) are stable and regions where two or more of them coexist. There are two crucial factors for the success of the CALPHAD method. The first factor is to find realistic as well as convenient mathematical models for the Gibbs energy for each phase. The second crucial factor is the availability of computer software for calculating equilibrium and various kinds of diagrams and databases with the stored assessed information. Today there are only several commercial products on the market, so if some organization or individual want make their own phase diagram, they must purchase a lot for the databases from the companies mentioned before. Because of this, it is a great impediment for the application of the CALPHAD method.

\section{Curve Fitting Method}

The method of curve fitting is that inputting a phase diagram into a computer or picking some points (feature points) on the curve, and then constructing the fitting equation by computer and output. This method does not involve the complex thermodynamic equation, and debug output is relatively easy, it has realized the conversion from the phase diagram on paper to digital phase diagram, and the method we discussed in this paper is a kind of fitting method.

\section{ABSTRACTION OF THE PROBLEM}

The phase diagram we use now, which is supported by mass of experiment and the experimental data. Therefore, the amount of labor for drawing a phase diagram is a dig. This paper provides a new way to fit the phase diagram that meets the requirements by the computer graphics with less labor. In this paper, the Bezier curve selected for the experiment is cubic Bezier curve(shown in Fig. 2.

Bezier curves are widely used in computer graphics to model smooth curves. As the curve is completely contained in the convex hull of its control points, the points can be graphically displayed and used to manipulate the curve intuitively. Affine transformations such as translation and rotation can be applied on the curve by applying the respective transform on the control points of the curve.

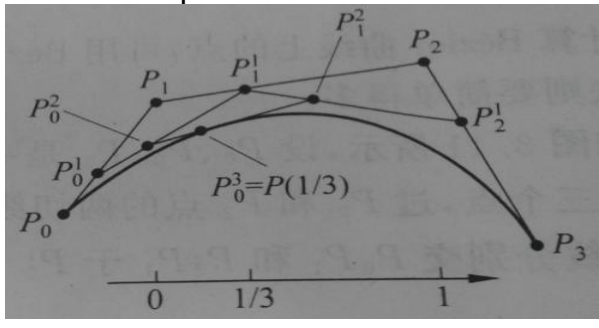

Figure 2. A cubic Bezier curve
Bezier curves are widely used in computer graphics to model smooth curves. As the curve is completely contained in the convex hull of its control points, the points can be graphically displayed and used to manipulate the curve intuitively. Affine transformations such as translation and rotation can be applied on the curve by applying the respective transform on the control points of the curve.

A Bezier curve is defined by a set of control points $\mathrm{P}_{0}$ through $\mathrm{P}_{\mathrm{n}}$, The first and last control points are always the end points of the curve; however, the intermediate control points (if any) generally do not lie on the curve. The Bezier curve formula (Equation (1 )) as follows:

$$
P(t)=\sum_{i=0}^{n} P_{i} B_{i, n}(t), t \in[0,1]
$$

The points $P_{i}$ are called control points for the Bezier curve. The polynomials (Equation (2)) are known as Bernstein basis polynomials of degree n.not "Eq. (1)" or "equation (1)", except at the beginning of a sentence: "Equation (1) is ..."

$$
B_{i, n}(t)=C_{n}^{i} t^{i}(1-t)^{n-i}=\frac{n !}{i !(n-i) !} t^{i} \bullet(1-t)^{n-i},(i=0,1, \cdots n)
$$

From the formulas above, we know that a cubic Bezier curve needs four control points to represent, so if we want to represent a phase diagram by Bezier curve we should find the control points of this phase diagram. Therefore, we can simply describe the question of reverse the control points as: given the points on the phase diagram, getting the $\square$ control points of this diagram, and ensure the accuracy of this method.

\section{SOLUTIONS TO THE PROBLEM}

\section{A. The Definition Method}

As mentioned above, the phase diagram with detailed data now is very precious, for the phase diagram with detailed data records, we can use the definition method to fitting it.

In order to get the control points of the Bezier curve, we need to construct the solution conditions, then we should introduce an algorithm, De Casteljau algorithm (3), De Casteljau algorithm is a recursive method to evaluate polynomials in Bernstein form or Bezier curves, named after its inventor Paul de Casteljau, which is the recursive algorithm of Bezier curve and can help us create solving equations.

$$
P_{i}^{k}=\left\{\begin{array}{l}
P_{i}, k=0 \\
(1-t) P_{i}^{k-1}+t P_{i+1}^{k-1}, k=1,2, \cdots n, i=0,1, \cdots n-k
\end{array} .\right.
$$

If we set the power of De Casteljau algorithm as 3, that $n$ equals 3. Simplify (3) we can get (4). $\mathrm{P}_{0}{ }^{3}$ represent the online point, $\mathrm{P}_{0}, \mathrm{P}_{1}, \mathrm{P}_{2}, \mathrm{P}_{3}$ represent the control points of Bezier curve. In this way, we just only need four points on the phase diagram, and then replace the corresponding parameters in (4) with those points, simultaneous, we can get the (5). 


$$
\begin{gathered}
P_{0}^{3}=(1-t)^{3} P_{0}+3 t(1-t)^{2} P_{1}+3 t^{2}(1-t) P_{2}+t^{3} P_{3} \\
\left\{\begin{array}{l}
P\left(t_{0}\right)=\left(1-t_{0}\right)^{3} P_{0}+3 t_{0}\left(1-t_{0}\right)^{2} P_{1}+3 t_{0}^{2}\left(1-t_{0}\right) P_{1}+t_{0}^{3} P_{3} \\
P\left(t_{1}\right)=\left(1-t_{1}\right)^{3} P_{0}+3 t_{0}\left(1-t_{1}\right)^{2} P_{1}+3 t_{1}^{2}\left(1-t_{1}\right) P_{1}+t_{1}^{3} P_{3} \\
P\left(t_{3}\right)=\left(1-t_{2}\right)^{3} P_{0}+3 t_{0}\left(1-t_{2}\right)^{2} P_{1}+3 t_{0}^{2}\left(1-t_{2}\right) P_{1}+t_{2}^{3} P_{3} \\
P\left(t_{3}\right)=\left(1-t_{3}\right)^{3} P_{0}+3 t_{0}\left(1-t_{3}\right)^{2} P_{1}+3 t_{3}^{2}\left(1-t_{3}\right) P_{1}+t_{3}^{3} P_{3}
\end{array}\right.
\end{gathered}
$$

We can see there is a parameter $t$ with the range $[0,1]$ in (4), when $\mathrm{t}=0, \mathrm{P}(0)=\mathrm{P}_{2}$, and when $\mathrm{t}=1, \mathrm{P}(1)=\mathrm{P}_{3}$, that is to say the start and the end point of Bezier curve is also its border control point. But when $0<t<1$, we don't know the relationship between a certain online point and it's corresponding parameter t. So we should find the relationship between them. As is shown in Fig.1, assuming the curve $\mathrm{P}_{0} \mathrm{Q}_{1} \mathrm{Q}_{2} \mathrm{P}_{3}$ is a phase diagram (just like the curve $\mathrm{C} 1$ in Fig.5), the points $\mathrm{Q}_{1}, \mathrm{Q}_{2}$, are two random curve points except the endpoints. The way to get the relationship between the certain online point and its corresponding parameter $t$ as follows:

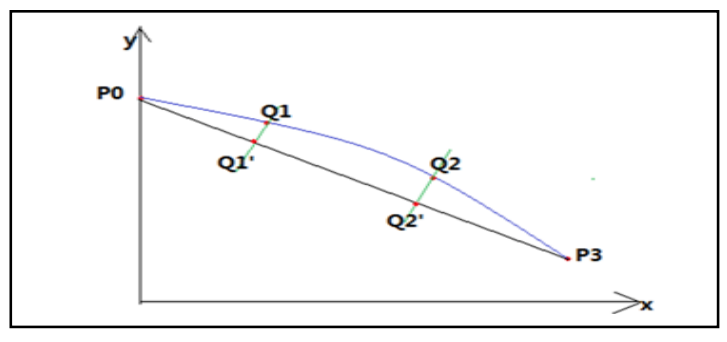

Figure 3. Auxiliary graph of calculating t

1) Modeling: Connecting the points $\mathrm{P} 0$ and $\mathrm{P} 3$, so that the segment P0P3 can be used as the boundary of the parameter $\mathrm{t}$. Because of the phase diagram we have observed are all the smooth curves, whose curvature is relatively small, we can take the segment $\mathrm{POP} 3$ as the boundary of the parameter $\mathrm{t}$, this conclusion can be confirmed by the error analysis below.

2) Drawing guideline: Making a perpendicular line to the segment $\mathrm{P} 0 \mathrm{P} 3$ though the points $\mathrm{Q}_{1}, \mathrm{Q}_{2}$, and we can get the vertical points $\mathrm{Q}_{1}, \mathrm{Q}_{2}$.

3) Replacing: The specific value of the length of segment $\mathrm{P}_{0} \mathrm{Q}_{1}, \mathrm{P}_{0} \mathrm{Q}_{2}$ and $\mathrm{P}_{0} \mathrm{P}_{3}$ can be as the substitute of the parameter $\mathrm{t}_{1}, \mathrm{t}_{2}$.

After having done the steps above, we replace the corresponding parameters in equations 5 with $t$ that have just calculated, and then we could get the control points of the phase diagram. At the same time we find that if we want represent a phase diagram we just only need four points on the curve. If we apply this method in the field of Materials and Metallurgy, we can get some data points that can't be achieved under the laboratory conditions by the points that achieved under the laboratory conditions. Thus, it can help the experimenter to set the condition of experiment and improve work efficiency.

\section{B. Plying-up Method}

The method introduced above aims at the phase diagram with detailed data to get the control points, and now we introduce another method for the diagram without detailed data.

As shown in Fig.5, it is an Al-Zn phase diagram. The abscissa represents the composition of the substance, and the ordinate represents the temperature [12]. There are only some endpoints data presented on the picture, whereas the other points data on curve could not get apparently. So we can use the steps to get the control points for those diagrams as follows:

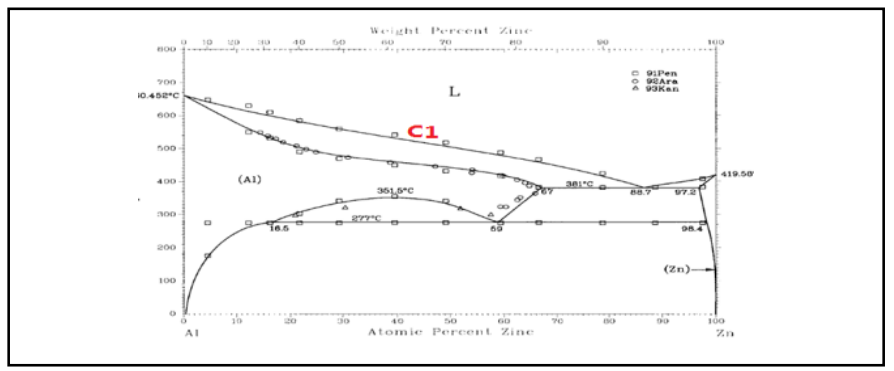

Figure 4. Al-Zn binary phase diagram

1) Setting background: Putting the photo of original phase diagram as the sketchpad (background) of drawing the new phase diagram.

2) Plying-up the coordinate system: That is to say, creating a new coordinate system. Firstly, we should select the origin for the new coordinate system, and of course the new origin should be coincided with the origin of the phase diagram. Secondly, drawing the coordinate. Obviously, it is not enough to make the two coordinates mutually perpendicular. Because most of the electronic phase diagram is generated by photos, the diagrams are easily distorted, so when we draw the coordinate, we should assure the coordinates are able to rotate, only in this way can we make sure that the new coordinate can be coincided with the phases diagram coordinate in maximum extent. Finally, adjusting the ratio of the coordinate system and consistent with the original coordinate system (the coordinate system are the green lines shown in Fig.5, and Fig.6 is the twice enlarged view of distract D in Fig.5).

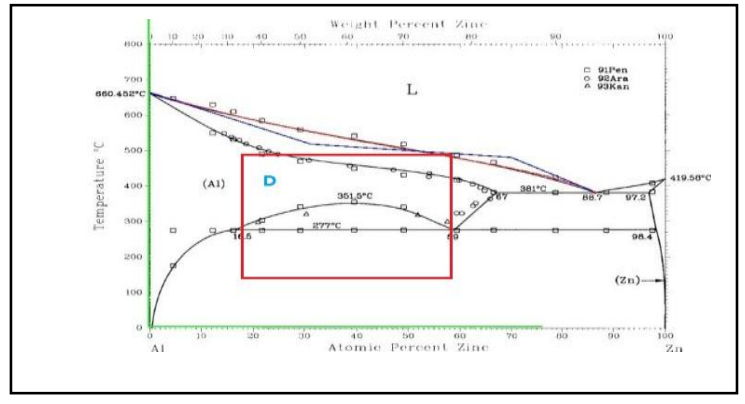

Figure 5. plying-up the coordinate system 


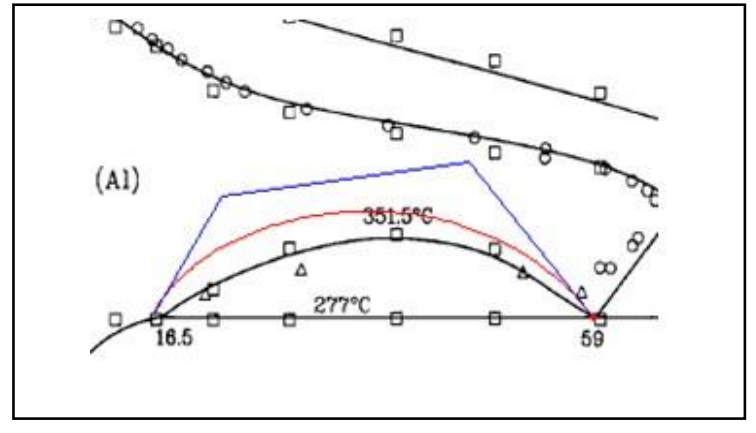

Figure 6. plying-up the phase diagram 1

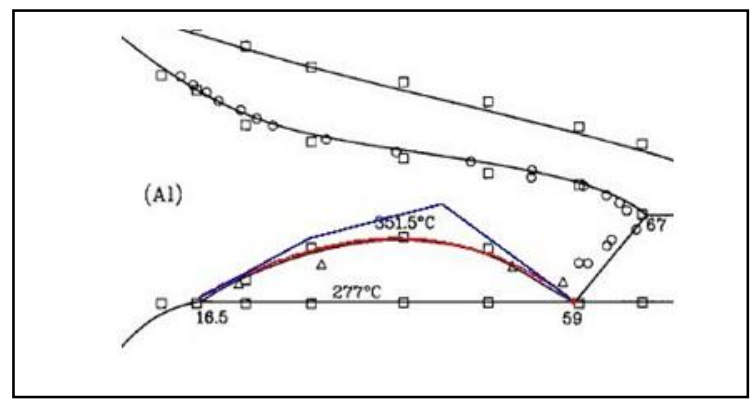

Figure 7. plying-up the phase diagram 2

3) Plying-up the phase diagram: As mentioned in the Definition Method, we just know the two border control points, what we do not know are the two middle control points. Now, how can we plying-up the diagram? Firstly, we choose any two middle control points, and then draw the curve (Fig.6 shows this situation, the blue polygon is the control points are connected end to end, the red curve represent the Bezier curve), it is obvious not what we want. Secondly, dragging the middle control points until the curve coincide with the phase diagram (Fig.7 shows the final result).

Recording result:Recording the data of control points.

All in all, we can use two methods of Bezier curve to represent and store the binary phase diagram.

\section{ANALYSIS OF THE EXPERIMENTAL RESULTS}

We have done several experiments to verify the feasibility of the above methods. The table 1 , table2, table 3 , are the results of error analysis, where the second column (original data) represents the data get from the authoritative books, and the third column (experimental data) represents the data get from either method above. Table 1 and Table 3 are the error analysis of the curve $\mathrm{C} 1$ (a binary phase diagram) in Fig.4, the Al-Zn binary phase diagram. Table 2 the error analysis of the curve $\mathrm{C} 2$ (another binary phase diagram) in Fig.8, the Bi-Ln binary phase diagram[14].

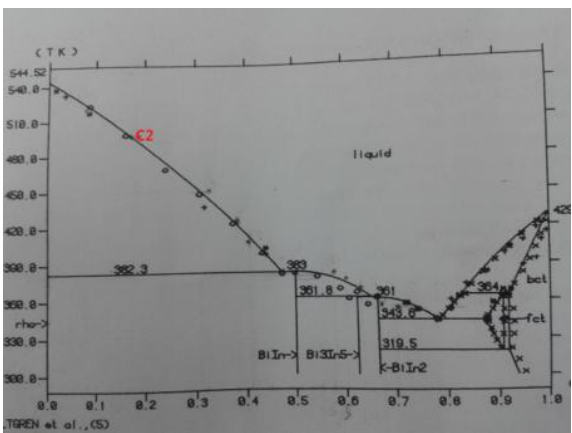

Figure $8 . \quad$ Bi-In binary phase diagram

In the area of metallurgy and material science, the composition of the material is the very important dimension, so we can use the composition of material as the standard of the error analysis.

The error analysis of Table 1 aims at the Definition Method, the Definition Method is applied to the phase diagram with original data, so we can confirm that the method of representing binary phase diagram by the Bezier curve is acceptable though the error analysis.

TABLE I. $\quad$ ERROR IN THE DEFINITION METHOD

\begin{tabular}{c|c|c|c|c}
\hline $\begin{array}{c}\text { Temperature } \\
\left({ }^{\mathbf{0}} \mathbf{C}\right)\end{array}$ & $\begin{array}{c}\text { Composition } \\
\text { original data) }\end{array}$ & $\begin{array}{c}\text { Composition } \\
\text { (experiment } \\
\text { data) }\end{array}$ & $\begin{array}{c}\text { Relative } \\
\text { error }\end{array}$ & $\begin{array}{c}\text { Absolute } \\
\text { erorr }\end{array}$ \\
\hline 600 & 0.000 & 0.000 & 0.000 & 0 \\
550 & 0.121 & 0.127 & 0.006 & $4.96 \%$ \\
530 & 0.161 & 0.164 & 0.003 & $1.83 \%$ \\
489 & 0.219 & 0.26 & 0.041 & $18.74 \%$ \\
470 & 0.292 & 0.317 & 0.025 & $8.55 \%$ \\
451 & 0.391 & 0.394 & 0.003 & $0.78 \%$ \\
428 & 0.491 & 0.499 & 0.008 & $0.16 \%$ \\
413 & 0.597 & 0.565 & -0.032 & $-5.33 \%$ \\
381 & 0.670 & 0.670 & 0.000 & 0 \\
\hline
\end{tabular}

TABLE II. ERROR IN PICKING POINTS OF THE PLYING-UP METHOD

\begin{tabular}{c|c|c|c|c}
\hline $\begin{array}{c}\text { Temperature } \\
\left({ }^{\mathbf{}} \mathbf{C} \text { ) }\right.\end{array}$ & $\begin{array}{c}\text { Composition } \\
\text { (original data) }\end{array}$ & $\begin{array}{c}\text { Composition } \\
\text { (experiment } \\
\text { data) }\end{array}$ & $\begin{array}{c}\text { Relative } \\
\text { error }\end{array}$ & $\begin{array}{c}\text { Absolute } \\
\text { error }\end{array}$ \\
\hline 541.23 & 0.000 & 0.000 & 0.000 & 0 \\
522.59 & 0.093 & 0.076 & -0.017 & $-18.31 \%$ \\
495.62 & 0.167 & 0.169 & 0.002 & $1.23 \%$ \\
450.00 & 0.308 & 0.321 & 0.013 & $4.24 \%$ \\
428.65 & 0.378 & 0.381 & 0.003 & $0.74 \%$ \\
412.49 & 0.443 & 0.416 & -0.027 & $-6.02 \%$ \\
382.30 & 0.475 & 0.475 & 0.000 & 0 \\
\hline
\end{tabular}

TABLE III. ERROR IN PLYING-UP METHOD

\begin{tabular}{c|c|c|c|c}
\hline $\begin{array}{c}\text { Temperatue } \\
\left({ }^{\mathbf{}} \mathbf{C}\right)\end{array}$ & $\begin{array}{c}\text { Composition } \\
\text { (original data) }\end{array}$ & $\begin{array}{c}\text { Composition } \\
\text { (experiment } \\
\text { data) }\end{array}$ & $\begin{array}{c}\text { Relative } \\
\text { error }\end{array}$ & Absolute error \\
\hline 660 & 0.000 & 0.000 & 0.000 & 0 \\
530 & 0.161 & 0.166 & 0.005 & $3.23 \%$ \\
489 & 0.219 & 0.255 & 0.036 & $1.65 \%$ \\
470 & 0.292 & 0.316 & 0.024 & $8.46 \%$ \\
451 & 0.391 & 0.413 & 0.022 & $5.75 \%$ \\
428 & 0.491 & 0.548 & 0.057 & $11.67 \%$ \\
413 & 0.597 & 0.600 & 0.003 & $0.60 \%$ \\
381 & 0.670 & 0.670 & 0.000 & 0 \\
\hline
\end{tabular}


The table 2 describes the error between the point data getting from the program and the original data of phase diagram in Plying-up Method. In order to proving Plying-up Method is right, we should use the phase diagram with original data even though the Plying-up Method is applied for the phase diagram without the original data.

The table 3 indicated the comparison between the curve generated by the Plying-up Method and the original phase diagram.

\section{CONCLUSION}

The proposed methods for representing the binary phase diagram by the Bezier curve in this paper, which is based on the understandable principle and is easy to realize. After having done the error analysis, we can see the results get by the Definition Method (the definition of Bezier curve), whose maximum error is $18 \%$, and the minimum error is $0.7 \%$, the overall error is within $5 \%$, and this confirm that the way to represent phase diagram by the Bezier curve is feasible. And then, because of there are less binary phase diagram with original data available, the Plying-up Method can be extended for the diagram without original data, and the overall absolute error of this method is within $5 \%$, in summary this method is feasible.

\section{ACKNOWLEDGMENT}

This work has been supported by grants from the Major Research plan Program of the National Natural Science Foundation of China (Grant No.51090381) and the 973 plan Program of National (Grant No. 2013CB632601).

\section{REFERENCES}

[1] Guofa Chen, Yungang Li. The principle of phase diagram and metallurgical phase diagrams. Metallurgical Industry Press. 2002

[2] Shengbi Zhang, Daozi Li. Phase diagram — principle, calculation and the application in Metallurgy. Bei Jing: Metallurgical Industry Press. 1986.

[3] Qingcheng Wei. Metallurgical Thermodynamics, Chong Qing: Chongqing University press, 1996.

[4] Wenchao Li. Metallurgical Thermodynamics. Bei Jing: Metallurgical Industry Press. 1995.

[5] Pingying Liu, Jingkang Guo. Overview the main methods for calculating phase diagram by the thermodynamic functions. Chinese ceramics. 2003,39(5):8.

[6] Jiaguang Sun, Shimin Hu. Computer Graphics. Tsinghua University press, 2009.

[7] Yunhe Pan. The methods and application of Computer Graphics. Bei Jing: Higher Education Press, 2003.

[8] Qingfang He. Computer graphics principles and algorithms tutorial. Bei Jing: Tsinghua University Press, 2010

[9] Rongxi Tang, JiaYe Wang, QunSheng Peng. Course of Computer Graphics. Bei Jing: Science Press. 2000

[10] Kaufman L, Bemstain H. Computer Calculation of Phase Diagram [M]. New York Academ ic Press. 1970: 1-173.

[11] Saunders N, Miodownik A P. CALPHAD (Calculation of phase diagrams) A comprehensive guide $[\mathrm{M}]$. Great Britain: Elsevier Science Ltd. 1998

[12] H. Okamoto (1995). Supplemental Literature Review. Journal of Phase Equilibria Vol. 16 NO. 31995

[13] Jiaxiang Chen. Handbook of steelmaking chart data. Metallurgical Industry Press. 2010 